\title{
An Exertion to Alleviate Stitch Defects During Garments Production
}

\author{
Mst. Farzana Sultana, Lecturer \\ Tahmina Akhter, Lecturer \\ Jaglul Hoque Mridha, Lecturer \\ Department of Textile Engineering, Northern University Bangladesh
}

Doi:10.19044/esj.2019.v15n36p489 URL:http://dx.doi.org/10.19044/esj.2019.v15n36p489

\begin{abstract}
In garments industry sewing is the most critical phase during an apparel production. Different types of sewing and stitching defects are occured in this phase due to various problems. As today's world each customer is expecting a very high quality garments with product variety, it has become a very challenging task for garments quality management. Here all the data were collected from Gardenia Wears Limited situated at Barmi- Sreepur, Gazipur, Dhaka and data were analyzed for reducing Defects per Hundred Unit (DHU\%) and also top 10 stitch defects were identified and analyzed later. Finally the work shows reduction of DHU\% from $5.23 \%$ to $3.48 \%$ and also reduce the top ten stitch defects with comparison to before trial and after trial data of ten days and it is proved that an industry can achieve higher production capability \& profitability with improved quality product and also saving cost due to reducing DHU\%.
\end{abstract}

Keyword: Sewing defects, Stitch defects, DHU\%

\section{Introduction}

In garments industry after completion of a shipment some garments are found to be rejected by the manufacturers. As they think that garments are soft goods and it will be non-repairable defects if low quality raw materials are used or faulty processes are operated or for employee casual behavior. So some check points should be kept in factory to control such issues. As a lot of garments are rejected after shipments, there is no over-night solution that can minimize the rejection percentage. Most of the organizations identified these garments rejected as these garments can't be used by any means. Reworking on garments is common but it hampers company production rate. If any industry decides to work on rejected garment pieces before delivery, it can't be possible because of not only hampering in smooth production rate but also 
focusing on rejected poor quality products that leaves a bad impact on factory economy as a whole (Juran J.M et al, 2008; Montgomery D.C., 2009; Dean J.W et al, 1994; Glock R.E et al, 2009). Every order is unique. But through these kind of rework it can be shown the ways to handling such problems \& bring down the rejection rate to minimum. For quality and productivity improvement rework is a must. Garments defects is a vital cause that can affect both manufacturer and purchaser if it can be possible to control defects rate during various activities in sewing departments through a proper supervision and strict operation condition (Pritesh Kankariya et al, 2009; Juran J.M et al, 2008). Through this study it is possible to know the way of handling the issues related to garments defects, to bring down the rejection rate of garments to minimum by reducing DHU\%, to produce a large no. of good quality products for minimization of DHU\%, to save cost \& to control defects by taking various actions in sewing department. Due to improper material handling during stitching, using defective feed mechanism, improper tension on thread, using blunt needle point $\&$ bent or damaged needle etc problems of different types of sewing \& stitching defects are found during production such as skipped stitch, staggered stitch, broken stitch, uneven stitch, seam puckering, raw edge, variable stitch density etc. To rectify $\&$ minimize defects at first it is important to know the defects mentioned below:

* Skipped stitch/Drop stitch/Broken stitch: In a successive stitches if one or more stitches fail in connecting the upper thread with the lower thread then it is called skipped stitch. Because of having $\mathrm{m} / \mathrm{c}$ problems during stitching sometimes such type of defects may happen but rework is possible for removing defects in order to improve product quality (Pranjali Chandurkar et al, 2017).

* Raw edge/frayed seam: If the tail end remains with the seam then it is called frayed seam which will cause a bad appearance. That's why it is considered as defective.

* Uneven stitch: When an operator stitches, if it becomes loose or wavy instead of straight, it may cause uneven stitch. It is occurred for variation of fabric properties, improper function during wear of garments (Dean J.W et al, 1994).

* Seam puckering: During stitching due to unequal stretch on the plies of fabric, fabric dimensional stability, extension in sewing thread, sewing thread shrinkage, undesirable \& uneven surface or gathering of fabric are seen on garments which is called seam puckering. As it destroys the appearance \& function of garments it is considered as defective (Md. Islam M. et al, 2016).. 


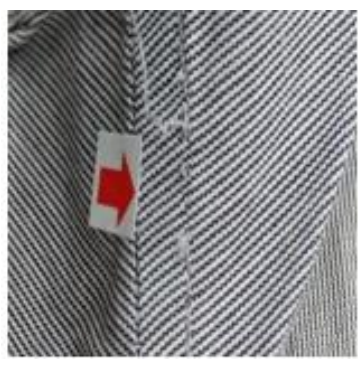

Broken stitch

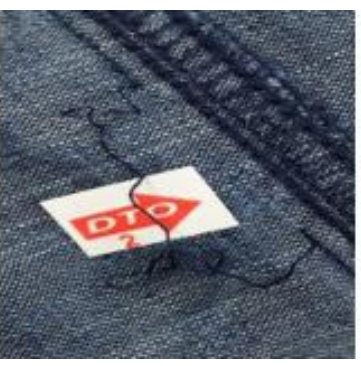

open seam

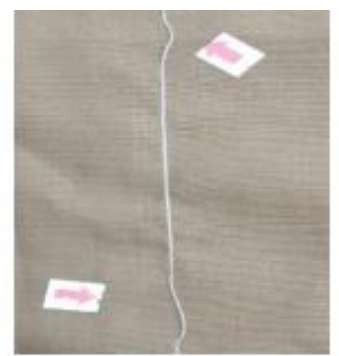

skipped stitch

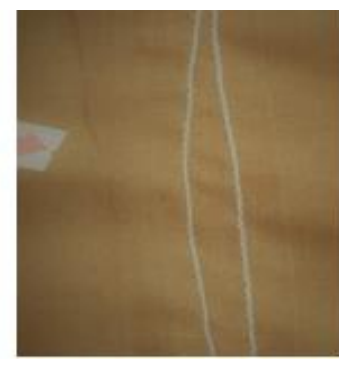

uneven stitch

\section{Material \& Method:}

\subsection{Material}

For executing the method following buyer's item were selected for analysis to reduce $\mathrm{DHU} \%$.

Buyer: TOTTUS

Style no: V20JHBERPRINT

Suppliers: Mahmud Denim Mills Ltd.

Size: 28, 30, 32, 34, 36, 38

Shade: COMBO-1, 2, 3, 4, 5, 6, 7, 8(AOP)
Fabric type: $100 \%$ cotton twill SAM: 2.39

No. of operation: 25

Fabric GSM: 185

Mixing: 60/40 cotton fabric

\subsection{Method:}

For experimental work at first needed to select a style that are running on sewing floor for production. 10 days data were collected before trial of minimizing DHU\% by noting down the total inspected pieces, total rejected pieces. Then from the data it were also identified the top ten defects that are happening during sewing of the preselected style. After that some corrective actions were taken to analyze whether it was possible to minimize the DHU\% or not by changing sewing $\mathrm{m} / \mathrm{c}$ setting such as resetting tensioner, time synchronization during stitch formation, awareness of operator about the physical properties of fabrics, proper lubrication on thread and machine, proper adjustment on feeding mechanism, using proper needle, needle point \& good quality sewing thread, adjustment on sewing thread tension etc. Then after trial again 10 days data taken by collecting the information of total inspected pieces \& total rejected pieces. Again the data for top 10 stitching defects noted down after trial. Finally the result between 10 days data of DHU\% on before trial with the DHU\% of after trial were compared. It is also showed the difference between top 10 stitch defects before trial with the after trial. Below a table is given on the causes $\&$ corrective actions taken to reduce defects during production based on different stitch varieties. 
Table 2.1: Causes \& corrective actions taken during production to reduce defects

\begin{tabular}{|l|l|}
\hline \multicolumn{1}{|c|}{ Causes of stitch defects } & \multicolumn{1}{|c|}{ Corrective actions taken } \\
\hline $\begin{array}{l}\text { 1. Improper time synchronization } \\
\text { between needle \& hook for loop } \\
\text { formation. }\end{array}$ & $\begin{array}{l}\text { 1. M/c settings were changed with } \\
\text { proper timing between needle and } \\
\text { hook. }\end{array}$ \\
\hline $\begin{array}{l}\text { 2. Irregular thread tension due to } \\
\text { loose or tight thread. }\end{array}$ & $\begin{array}{l}\text {. Thread tension were properly } \\
\text { adjusted during stitching. }\end{array}$ \\
\hline 3. Fabric flagging during sewing. & $\begin{array}{l}\text { 3. Adjusting the pressure of presser } \\
\text { foot. }\end{array}$ \\
\hline $\begin{array}{l}\text { 4. Thread balance way was } \\
\text { improper. }\end{array}$ & $\begin{array}{l}\text { 4. Bobbin thread tension way were } \\
\text { properly adjusted. }\end{array}$ \\
\hline $\begin{array}{l}\text { 5. For needle deflection. } \\
\text { 6. Defective motion of feed dog. } \\
\text { correct needle point. }\end{array}$ & 6. Proper adjustment of feed dog. \\
\hline $\begin{array}{l}\text { 7. Using sewing thread without } \\
\text { lubrication. }\end{array}$ & $\begin{array}{l}\text { 7. Proper lubrication were given on } \\
\text { sewing thread. }\end{array}$ \\
\hline 8. Needle was heated too much. & 8. Using needle lubricant. \\
\hline
\end{tabular}

\section{Result \& Discussion:}

\subsection{Experimental Data (10 days DHU\% Report before trial):}

Table 3.1: Day wise DHU\% Report before Trial

\begin{tabular}{|c|c|c|c|c|}
\hline \multicolumn{5}{|c|}{ Style- V20JHBERPRINT } \\
\hline $\begin{array}{c}\text { Serial } \\
\text { No }\end{array}$ & $\begin{array}{c}\text { No. Of } \\
\text { Days }\end{array}$ & $\begin{array}{c}\text { Total No. Of } \\
\text { Defects }\end{array}$ & $\begin{array}{c}\text { Total Check } \\
\text { Points }\end{array}$ & Total DHU\% \\
\hline 1. & Day 1 & 195 & 3000 & $6.50 \%$ \\
\hline 2. & Day 2 & 149 & 3050 & $4.88 \%$ \\
\hline 3. & Day 3 & 160 & 2984 & $5.36 \%$ \\
\hline 4. & Day 4 & 146 & 3140 & $4.64 \%$ \\
\hline 5. & Day 5 & 161 & 2700 & $5.96 \%$ \\
\hline 6. & Day 6 & 154 & 2789 & $5.52 \%$ \\
\hline 7. & Day 7 & 151 & 2900 & $5.20 \%$ \\
\hline 8. & Day 8 & 145 & 3100 & $4.67 \%$ \\
\hline 9. & Day 9 & 112 & 2450 & $4.57 \%$ \\
\hline 10. & Day 10 & 144 & 2890 & $4.98 \%$ \\
\hline & & $\begin{array}{c}\text { Total } \\
\text { defects=1517 }\end{array}$ & $\begin{array}{c}\text { Total checked } \\
\text { pieces=29003 }\end{array}$ \\
\hline
\end{tabular}

Now, Defects per hundred units

Total no. of defects

Total no. of inspected pieces

1517

29003

$$
=\times 100
$$

$=5.23 \%$ 


\subsection{Experimental Data (10 days DHU\% Report after trial):}

Table 3.2: Day wise DHU\% report after trial

\begin{tabular}{|c|c|c|c|c|}
\hline \multicolumn{5}{|c|}{ Style: V20JHBERPRINT } \\
\hline $\begin{array}{c}\text { Serial } \\
\text { no }\end{array}$ & No. of Days & $\begin{array}{l}\text { Total no. of } \\
\text { Defects }\end{array}$ & $\begin{array}{c}\text { Total Check } \\
\text { points }\end{array}$ & Total DHU\% \\
\hline 1. & Days 1 & 129 & 2950 & $4.37 \%$ \\
\hline 2. & Days 2 & 119 & 2800 & $4.25 \%$ \\
\hline 3. & Days 3 & 105 & 2770 & $3.79 \%$ \\
\hline 4. & Days 4 & 132 & 3250 & $4.06 \%$ \\
\hline 5. & Days 5 & 111 & 3100 & $3.58 \%$ \\
\hline 6. & Days 6 & 105 & 3000 & $3.50 \%$ \\
\hline 7. & Days 7 & 125 & 2900 & $4.31 \%$ \\
\hline 8. & Days 8 & 121 & 3300 & $3.66 \%$ \\
\hline 9. & Days 9 & 68 & 3500 & $1.94 \%$ \\
\hline 10. & Days 10 & 65 & 3427 & $1.89 \%$ \\
\hline & & Total defects= & $\begin{array}{l}\text { Total Checked } \\
\text { pieces= 30997 }\end{array}$ \\
\hline
\end{tabular}

Now, Defects per hundred units

Total no. of defects

$$
=\longdiv { \text { Totalno. } } \times 1 0 0
$$

Total no. of inspected pieces

$$
1080
$$

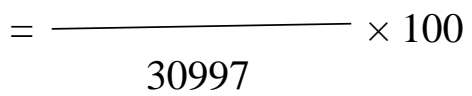

$$
=3.48 \%
$$

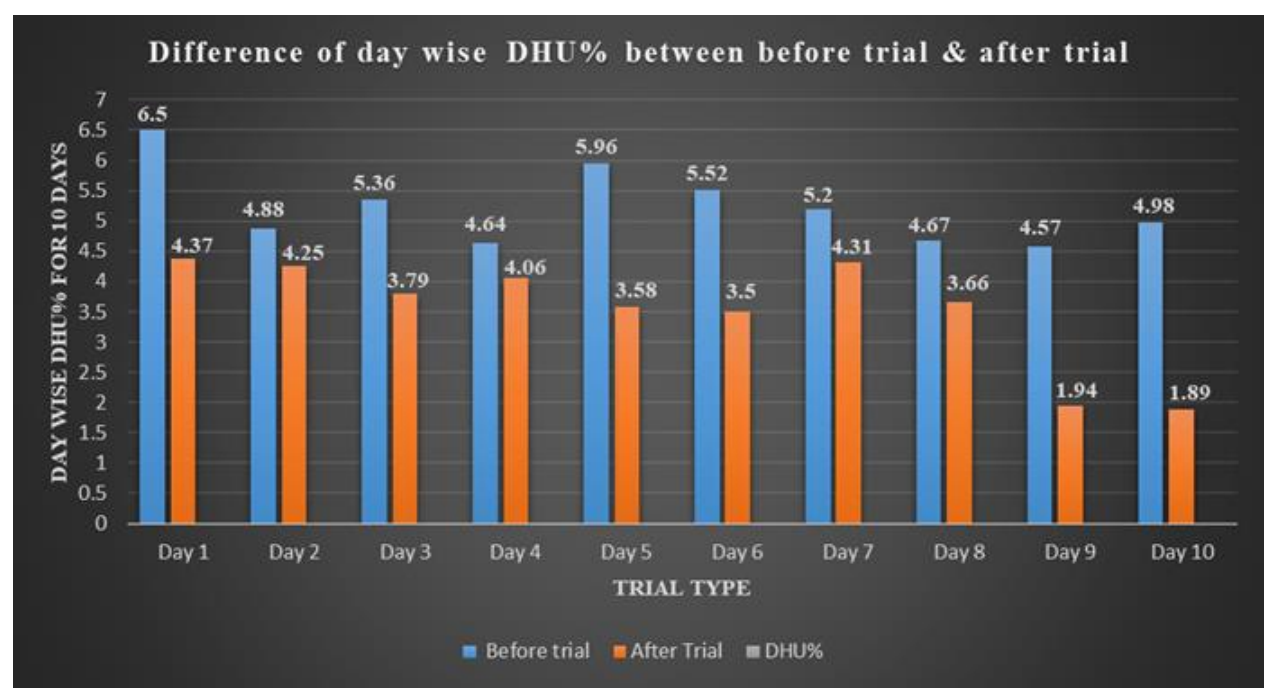

Figure 3.1: Chart on difference of day wise DHU\% between 10 days of before trail and 10 days of after trial 
The chart shows day wise DHU\% report before trial \& after trial for 10 days. It also shows that the highest DHU\% before trial was $6.5 \%$ \& lowest $4.57 \%$ whereas after trial highest DHU\% was $4.37 \%$ \& lowest $1.89 \%$.

\subsection{Experimental data (Day to Day data For Top Ten Stitch Defects for 10 days Before Trail):}

Table 4.3: Defects/day For Top 10 Stitch Defects before Trial

\begin{tabular}{|l|l|l|l|l|l|l|l|l|l|l|l|l|}
\hline $\begin{array}{l}\text { Serial } \\
\text { no. }\end{array}$ & Defects/Day & $\mathbf{1}$ & $\mathbf{2}$ & $\mathbf{3}$ & $\mathbf{4}$ & $\mathbf{5}$ & $\mathbf{6}$ & $\mathbf{7}$ & $\mathbf{8}$ & $\mathbf{9}$ & $\mathbf{1 0}$ & Total \\
\hline 1. & Skipped stitch & 27 & 25 & 20 & 14 & 13 & 12 & 13 & 12 & 12 & 12 & 160 \\
\hline 2. & Broken stitch & 21 & 17 & 19 & 17 & 19 & 13 & 11 & 7 & 13 & 10 & 147 \\
\hline 3. & Puckering & 13 & 15 & 17 & 18 & 15 & 19 & 12 & 9 & 11 & 13 & 142 \\
\hline 4. & Uneven stitch & 21 & 9 & 12 & 10 & 10 & 7 & 14 & 15 & 9 & 11 & 118 \\
\hline 5. & Measurement & 23 & 21 & 22 & 16 & 22 & 17 & 13 & 10 & 15 & 20 & 179 \\
\hline 6. & $\begin{array}{l}\text { Bar tack } \\
\text { missing }\end{array}$ & 16 & 15 & 13 & 15 & 14 & 13 & 16 & 16 & 13 & 12 & 143 \\
\hline 7. & Short stitch & 20 & 15 & 16 & 14 & 15 & 14 & 12 & 10 & 10 & 11 & 137 \\
\hline 8. & Join stitch & 23 & 20 & 18 & 19 & 17 & 15 & 15 & 14 & 15 & 14 & 170 \\
\hline 9. & Up \& Down & 10 & 12 & 12 & 10 & 13 & 11 & 14 & 12 & 10 & 11 & 115 \\
\hline 10. & Others & 26 & 29 & 27 & 33 & 29 & 24 & 22 & 23 & 22 & 29 & 264 \\
\hline
\end{tabular}

3.4 Experimental data (Day to Day data For Top Ten Stitch Defects for 10 days After Trail):

Table 4.4: Defects/day For Top 10 Stitch Defects after Trial

\begin{tabular}{|l|l|l|l|l|l|l|l|l|l|l|l|l|}
\hline $\begin{array}{l}\text { Serial } \\
\text { no. }\end{array}$ & Defects/Day & $\mathbf{1}$ & $\mathbf{2}$ & $\mathbf{3}$ & $\mathbf{4}$ & $\mathbf{5}$ & $\mathbf{6}$ & $\mathbf{7}$ & $\mathbf{8}$ & $\mathbf{9}$ & $\mathbf{1 0}$ & Total \\
\hline 1. & Skipped stitch & 11 & 12 & 7 & 5 & 6 & 10 & 7 & 9 & 7 & 3 & 77 \\
\hline 2. & Broken stitch & 16 & 16 & 15 & 13 & 16 & 16 & 8 & 9 & 9 & 7 & 125 \\
\hline 3. & Puckering & 13 & 15 & 12 & 11 & 9 & 11 & 14 & 10 & 4 & 5 & 104 \\
\hline 4. & Uneven stitch & 11 & 9 & 12 & 9 & 4 & 9 & 10 & 13 & 10 & 8 & 95 \\
\hline 5. & Measurement & 20 & 18 & 14 & 17 & 15 & 17 & 13 & 6 & 6 & 7 & 133 \\
\hline 6. & $\begin{array}{l}\text { Bar tack } \\
\text { missing }\end{array}$ & 9 & 12 & 3 & 9 & 9 & 7 & 5 & 4 & 7 & 2 & 67 \\
\hline 7. & Short stitch & 10 & 11 & 7 & 6 & 5 & 10 & 7 & 4 & 2 & 4 & 66 \\
\hline 8. & Join stitch & 12 & 12 & 10 & 11 & 6 & 7 & 5 & 9 & 6 & 5 & 83 \\
\hline 9. & Up \& Down & 7 & 9 & 8 & 5 & 10 & 8 & 3 & 3 & 5 & 3 & 61 \\
\hline 10. & Others & 21 & 20 & 19 & 16 & 18 & 14 & 17 & 17 & 19 & 14 & 175 \\
\hline
\end{tabular}




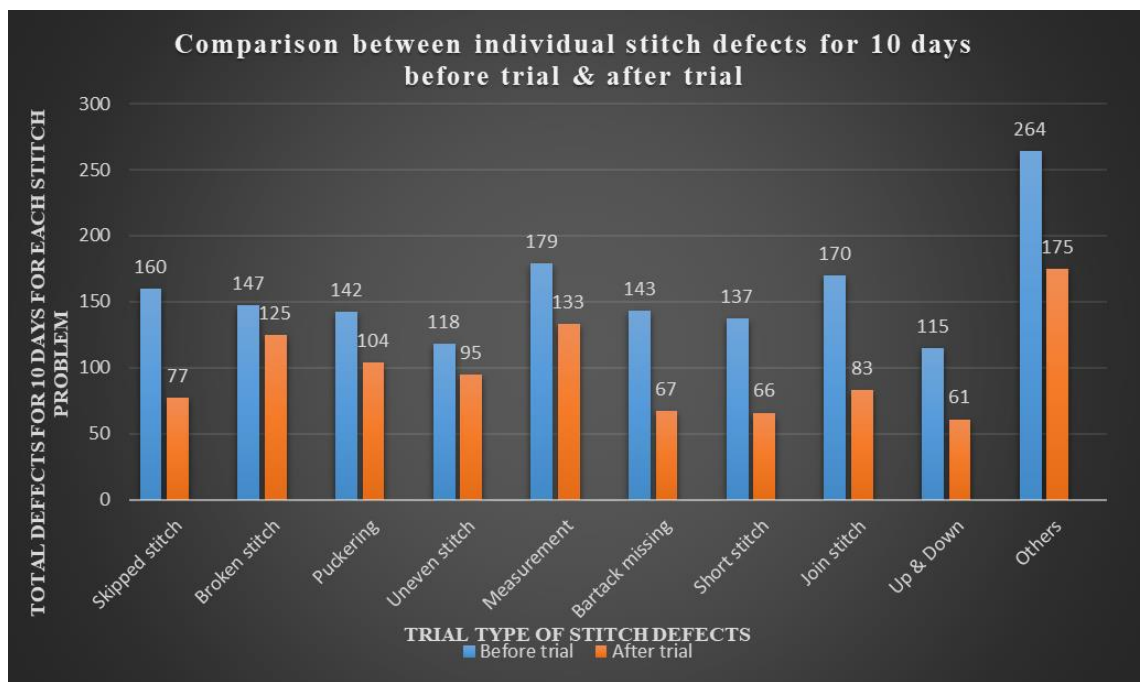

Figure 3.2: Chart on difference of total defects for each stitch defect between before trail data and after trial data

The chart shows the stitch defects in total for 10 days individually before trial \& after trail. It presents that each defects minimized individually in comparison with before trail \& after trail. The chart shows for stitch defects like skipped stitch, broken stitch, puckering, uneven stitch, measurement, bar tack missing, short stitch, join stitch, up \& down, others before trial value were respectively $160,147,142,118,179,143,147,170,115,264$ whereas after trial were respectively 77, 125, 104, 95, 133, 67, 66, 83, 61, 175.

Figure 3.3: Chart on difference between total DHU\% before trail data and after trial data

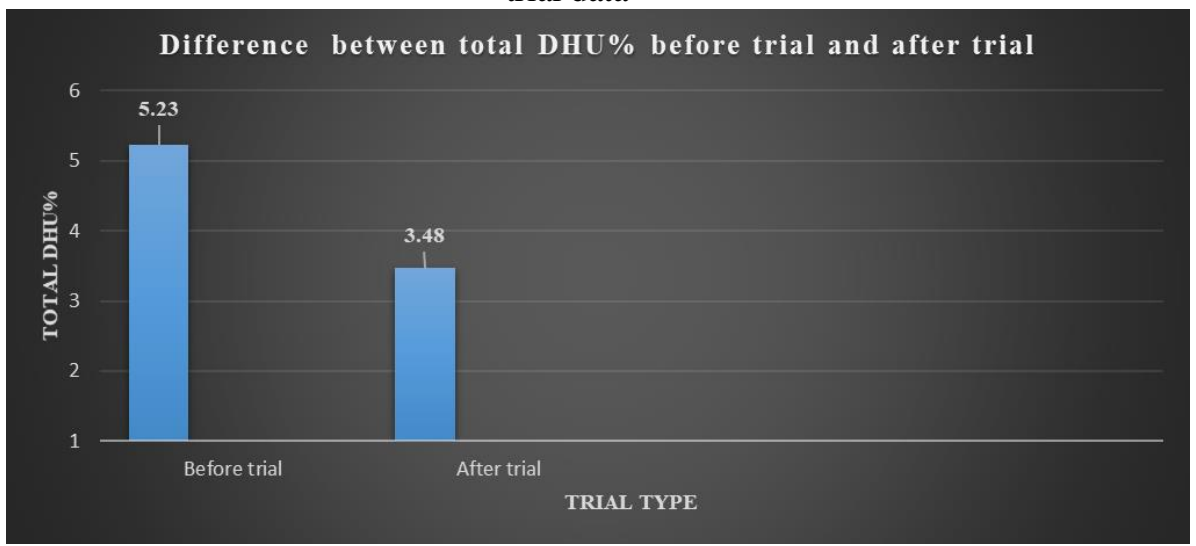

The chart represents the difference between total DHU\% for 10 days before trial and after trial. It shows that the total DHU\% before trial was 5.23\% whereas after trail was $3.48 \%$. 


\subsection{Experimental Result:}

1. Reduction of DHU\% like before trial DHU\% for 10 days were $5.23 \%$ \& after trial were $3.48 \%$.

2. Reduction of top ten stitch defects individually such as skipped stitch, broken stitch, puckering, uneven stitch, measurement, bar tack missing, short stitch, join stitch, up \& down, others before trial value were respectively 160 , $147,142,118,179,143,147,170,115,264$ whereas after trial value were respectively $77,125,104,95,133,67,66,83,61,175$.

3. DHU\% decreases from $5.23 \%$ to $3.48 \%$ that means it decreases $1.75 \%$ from before trial value.

\section{Conclusion}

It can be concluded that by taking various actions including setting tensioner of $\mathrm{m} / \mathrm{c}$, proper lubrication of $\mathrm{m} / \mathrm{c}$ and threads, time synchronization, skilled operators, consciousness of operators about physical properties of fabric, proper handling of material etc. it is possible to bring down the DHU\%. The result shows that the total DHU\% for 10 days before trial was $5.23 \%$ and after trial total DHU\% for 10 days is $3.48 \%$ which is less than before trial value. But these results can be even better if corrective actions are taken with consciousness and carefulness.

\section{References:}

1. Juran J.M., Gryna F.M., Quality Planning \& Analysis: For Enterprise Quality, Edition 2008, Tata McGraw-Hill Publication.

2. Montgomery D.C., Introduction to Statistical Quality Control, Edition 2009, John Wiley and Sons, Inc. Publication.

3. Dean J.W. and Bowen D.E., Management Theory and Total Quality: Improving Research and Practice and Theory Development, the Academy of Management Review, Vol. 19, Issue 3, 1994, 392-418.

4. Glock R.E., Kunz G.I., Apparel Manufacturing: Sewn Product analysis, $4^{\text {th }}$ Edition, Pearson Publication.

5. Pritesh Kankariya, KeshavValase, Performance improvement in Garment industries by reducing defects using six sigma methodologies, International Journal of Scientific Research Engineering \& Technology Vol. 6(3), 2017, 228-236.

6. Pranjali Chandurkar, Madhuri Kakde, Chitra Patil, Minimization of Defects in Knitted Fabric, International Journal on Textile Engineering and Processes, Vol. 2(3), 2016, 13-18.

7. Md. Islam M., Khan A.M., Md. Khan M.R., Minimization of Reworks in Quality and Productivity Improvement, The Apparel Industry, International Journal of Engineering and Applied Sciences, Vol. 1(4), 2013, 147-16. 